\title{
Continuum contribution to excitonic four-wave mixing
}

Birkedal, Dan; Sayed, Karim EI; Vadim, Lyssenko; Hvam, Jørn Märcher

Published in:

Summaries of Papers Presented at the Quantum Electronics and Laser Science Conference

Link to article, DOI:

10.1109/QELS.1996.865629

Publication date:

1996

Document Version

Publisher's PDF, also known as Version of record

Link back to DTU Orbit

Citation (APA):

Birkedal, D., Sayed, K. E., Vadim, L., \& Hvam, J. M. (1996). Continuum contribution to excitonic four-wave mixing. In Summaries of Papers Presented at the Quantum Electronics and Laser Science Conference (pp. 107107). IEEE. https://doi.org/10.1109/QELS.1996.865629

\section{General rights}

Copyright and moral rights for the publications made accessible in the public portal are retained by the authors and/or other copyright owners and it is a condition of accessing publications that users recognise and abide by the legal requirements associated with these rights.

- Users may download and print one copy of any publication from the public portal for the purpose of private study or research.

- You may not further distribute the material or use it for any profit-making activity or commercial gain

- You may freely distribute the URL identifying the publication in the public portal 


\section{QWB5}

9:15 am

Continuum contribution to excitonic fourwave mixing

D. Birkedal, K. E. Sayed, V. G. Lyssenko, J. M. Hvam, Mikroelektronik Centret, The Technical University of Denmark, DK-2800 Lyngby, Denmark

We present an experimental and theoretical investigation of ultrafast transient four-wave mixing (TFWM) of GaAs / AlGaAs quantum wells for simultaneous excitation of exciton and continuum states. Recent TFWM experiments on semiconductors have shown unexpected results when both discrete exciton states and continuum states have been excited simultaneously by ultrafast laser pulses. ${ }^{1,2}$ The decay of the signal, as a function of delay $\tau$, is significantly faster than the dephasing time of the excitons. However, the width of the spectrally resolved excitonic TFWM response and the real-time evolution of the TFWM signal is reflecting the instrinsic excitonic dephasing time.

We have investigated a $\mathrm{GaAs} / \mathrm{Al}_{0.3^{-}}$ $\mathrm{Ga}_{0.7} \mathrm{As}$ multiple-quantum-well sample with spectrally resolved TFWM. The

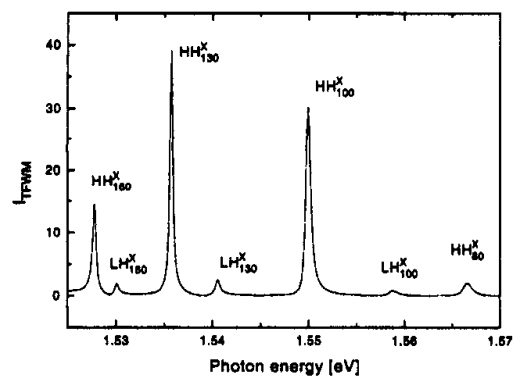

QWB5 Fig. 1 Transient four-wave mixing spectrum at zero time delay. The spectrum shows the heavy $(\mathrm{HH})$ and light hole (LH) excitonic resonances for the $160,130,100$, and $80 \AA$ quantum wells.

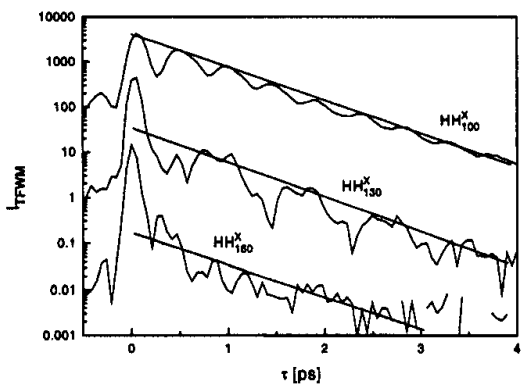

QWB5 Fig. 2 Intensity of the transient four-wave mixing signal at the heavy hole excitonic resonances of the 160,130 , and $100 \AA$ quantum wells. The curves have been displaced for clarity and the lines are guides for the eye. The fast initial component of the traces for the 160 and $130 \AA$ quantum wells is due to continuum excitation.
MBE-grown sample consists of wells of the thicknesses $80,100,130$, and $160 \AA$ and was kept at $5 \mathrm{~K}$ during the experiments. The sample was excited by $100-\mathrm{fs}$ pulses from a self-modelocked Ti:sapphire laser in the two-beam selfdiffraction geometry. ${ }^{3}$ The spectrum of the TFWM signal for zero time delay is shown in Fig. 1 for resonant excitation of the heavy hole exciton of the $100 \AA$ quantum well. The width of the excitonic resonances are similar to those observed in the absortion spectra of the sample $(<1$ meV), indicating dephasing times of several picoseconds. The 130 and $160 \AA$ quantum wells are excited several tens of $\mathrm{meV}$ into the continuum and the delay dependence of the excitonic TFWM intensities clearly reflects this. Figure 2 shows the intensity of the TFWM signal at the excitonic resonances as a function of delay. The response from the $100 \AA$ quantum well shows quantum beats associated with the light and heavy hole excitons and a decay of several picoseconds in agreement with the width of the resonance. The responses from the 130 and $160 \AA$ wells show a fast initial component of the order of the laser pulse followed by a slower component on the picosecond timescale. While the initial fast component is of the same magnitude as the response from the $100 \AA$ quantum well, the slow component is seen to be several orders of magnitude less intense.

We show that these observations are well explained using a model based on the semiconductor Bloch equations, taking populations generated by local fields into account. It is demonstrated that, for continuum excitation, the main signal at the spectral position of the exciton is due to diffraction in a second-order population grating of excitons generated by the action of the local field of the continuum states on the first order polarization of the exciton states. However, the local field of the continuum states is only significant within a time corresponding to the inverse width of the excitation. This is shown to result from interference of the first-order polarization of the continuum states.

1. J. Feldmann, T. Meier, G. von Plessen et al., Phys. Rev. Lett. 70, 3027 (1993).

2. U. Siegner, M.-A. Mycek, S. Glutsch, D. S. Chemla, Phys. Rev. Lett. 74, 470 (1995).

3. T. Yajima, Y. Taira, J. Phys. Soc. Jap. 47, 1620 (1979)

\section{QWB6}

9:30 am

Three-beam four-wave mixing on GaAs using 15-fs pulses

M. U. Wehner, D. Steinbach, M. Wegener, Institut für Angewandte Physik, Univ. Karlsruhe, D-76128 Karlsruhe, Germany

Two-beam four-wave mixing (FWM) experiments on bulk GaAs at low temperatures using pulses of $15 \mathrm{fs}$ in duration have recently revealed two observations: i) non-Markovian relaxation resulting from exciton-LO-phonon interaction ${ }^{1}$ at

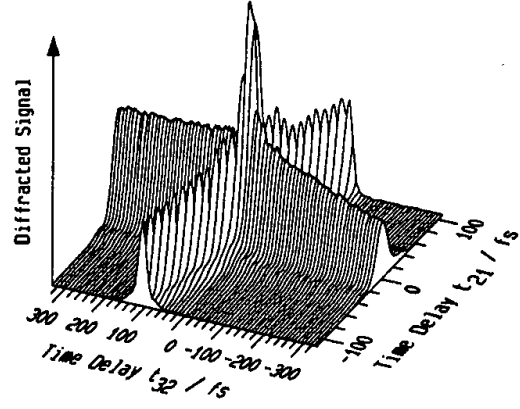

QWB6 Fig. 1 Experiment: Diffracted signal as a function of $t_{21}$ and $t_{32}, \mathrm{GaAs}$, $\mathrm{T}=77 \mathrm{~K}$.

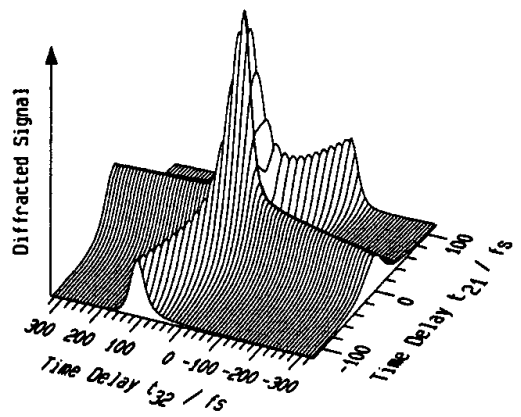

QWB6 Fig. 2 Model: Diffracted signal as a function of $t_{21}$ and $t_{32}$.

intermediate carrier densities $\left(\approx 10^{16}\right.$ $\mathrm{cm}^{-3}$ ) and ii) an apparent contradiction between the observed decay in the time delay domain and the FWM-linewidth ${ }^{2}$ at densities as low as a few $10^{14} \mathrm{~cm}^{-3}$. This apparent contradiction, which has previously only been observed on Fano-resonances, ${ }^{3}$ has had no explanation up to this point.

To further investigate these aspects we have performed three-beam experiments under identical conditions. The three 15-fs pulses, tuned to the band edge, with wave vectors $\vec{q}_{1}, \vec{q}_{2}$, and $\vec{q}_{3}$ arrive at times $t_{1}, t_{2}$, and $t_{3}$, respectively. The diffracted signal in direction $\vec{q}_{3}+\vec{q}_{2}-$ $\vec{q}_{1}$ is detected as a function of the time delays $t_{21}=t_{2}-t_{1}$ and $t_{32}=t_{3}-t_{2}$. Figure 1 shows the diffracted signal as a function of the two time delays $t_{21}$ and $t_{32}$ at rather low total carrier density $\left(9.5 \cdot 10^{14}\right.$ $\mathrm{cm}^{-3}$ ). The half width at half maximum of the FWM spectra (not shown) is $1 \mathrm{meV}$ (resolution limited), which corresponds to a dephasing time $T_{2}$ of $T_{2}>658 \mathrm{fs}$, which is in apparent contradiction with the rapid decay $\left(\approx 10 \mathrm{fs}\right.$ ) in the $t_{21}$ domain. However, the decay for $t_{21}=0$ and negative $t_{32}$ exhibits a time constant of $\approx 1$ $p s$, which is consistent with the observed linewidth. This behavior can neither be understood within the opitcal Bloch equations nor within the semiconductor Bloch equations together with a constant $T_{2}$. Here we present a model for the dominant nonlinear optical contribution under ultrafast excitation that accounts for the scattering of 1-s excitons with contin- 